\title{
Clinical evaluation of shoulder ROM in volleyball and handball players in youth categories
}

\author{
Petr Benda, Tereza Nováková*, Lenka Žáková
}

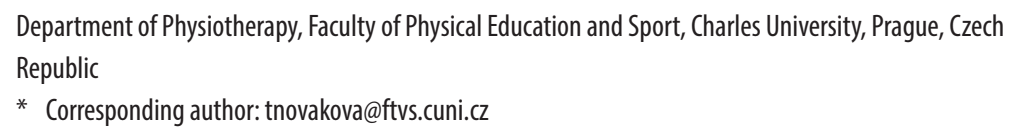

\begin{abstract}
Aims. The aim of this study was to clarify whether volleyball and handball players, as representatives of the so-called overhead athletes, already have an increased ROM and specific local hypermobility in the shoulder joints in the junior categories. These changes are described by goniometric measurements and hypermobility tests according to Sachse and Beighton. The results are examined with respect to gender and sport specialization.

Methods. Ninety-five subjects without previous shoulder joint injury aged 16 to 19 years, including 73 volleyball and handball players, were tested. The cohort included 33 men, 40 women, 34 handball players and 39 volleyball players. The control group consisted of 22 participants, including 11 men and 11 women. The majority $(90.5 \%)$ of the study participants were right-hand dominant. Only $9.5 \%$ of the participants were left-handed. Measurements were taken with a goniometer with digital display and hypermobility tests according to Sachse and Beighton.

Results. In a selected group of volleyball and handball players, the ROM of the shoulder joints of the dominant arm was significantly greater in three ways: into extension, horizontal adduction and external rotation. On the other hand, the ROM of their shoulder joints in internal rotation is smaller and the research group has significant hypermobility in the glenohumeral (scapulohumeral) joint compared to the control group. Our study showed that females compared to males playing volleyball and handball have a significantly higher degree of internal rotation of both dominant and non-dominant arms. No significant differences were found when comparing volleyball and handball players. The difference in ROM between the dominant and non-dominant arms of volleyball and handball players was shown in extension and external rotation.
\end{abstract}

\section{KEYWORDS}

goniometry; range of motion; hypermobility; handball; volleyball

DOI

10.14712/23366052.2021.11

(c) 2021 The Authors. This is an open-access article distributed under the terms of the Creative Commons Attribution License (http://creativecommons.org/licenses/by/4.0), which permits unrestricted use, distribution, and reproduction in any medium, provided the original author and source are credited. 


\section{INTRODUCTION}

Throwing above head level is an extremely complex and agile movement. The thrower's arm must be sufficiently lax to allow excessive external rotation, but at the same time stable enough to prevent subluxations of the humeral head, which requires a balance between joint mobility and functional stability. We speak of this as the "thrower's paradox". This balance is very often disturbed and is thought to lead to various types of injuries to surrounding tissues. Among these injuries, the authors also include "internal impingement", which was first described in 1992 by the Tennis Association. In total, patients with this pathology usually have full range of motion (ROM), but on the dominant arm the external rotation is increased by $10^{\circ}$ to $15^{\circ}$ and the internal rotation is decreased by $10^{\circ}$ to $15^{\circ}$ compared to the non-dominant arm (Wilk et al., 2011).

According to Wilk and Arrigo (1992), most overhead athletes show significant glenohumeral joint laxity (scapulohumeral), which allows for excessive ROM. The hypermobility of the "overhead shoulder" was referred to in this article as the "thrower's laxity".

However, Borsa and colleagues did not report any difference in overhead athletes compared to the control group when objectively testing joint hypermobility on a Telos device (Borsa et al., 2005).

An extensive study of the "overhead shoulders" of New York Yankees baseball players has come to several conclusions. The first conclusion was that players have an increased range of external rotation and a reduced range of internal rotation of the dominant arm, and thus an altered arc of glenohumeral movement in the throwing position relative to the non-dominant arm. In this study, the authors also sought to determine the relationship between a player's age and years of active career and ROM in the shoulder joints or hypermobility in the shoulder joints. According to their results, there is no significant relationship in this direction (Bigliani et al., 1997).

Regarding the shoulder joint and throw, the relationship between the individual's height and weight and the speed of the shoulder during the baseball throw was also observed. The result was negative, no regularity between these factors was demonstrated (Pappas, Zawacki, \& Sullivan, 1985).

\section{THEORETICAL BACKGROUND}

Jobe and Pink (1993) describe that overhead athletes most often have a rotator cuff rupture during a shoulder injury. These altered ranges of motion (increased in extension, external rotation, and decreased in internal rotation) can affect this type of injury.

Due to the nature of volleyball and handball, a significant burden is placed on external rotation. Wilk et al. (2011) described that there is a significant difference in the ROM between the external and internal rotators on the dominant shoulder joint. This author described that overhead athletes usually have a full ROM, but on the dominant arm the outer rotation is increased by $10^{\circ}$ to $15^{\circ}$ and the inner rotation is reduced by $10^{\circ}$ to $15^{\circ}$ compared to the non-dominant arm.

According to Russek (1999), 0.6-31.5\% of the adult population has hypermobility, depending on factors such as ethnicity or age. Studies in rheumatology clinics across Europe report a prevalence of joint hypermobility in adults of 5-15\%. 
Demographic characteristics by population are reported a lot, for example, hypermobility is more common in Asians than in Europeans. In our population, however, the true degree of generalised hypermobility is unknown. Janda states that it is $40 \%$ of the female population, but also adds that local pathological hypermobility is independant of gender (Janda, 2004).

In athletes, local pathological hypermobility is often present, which is further divided into two types. The first type is compensatory hypermobility in individual segments, compensation for reduced range in another segment. The second type is post-traumatic instability arising, for example, after injuries to the shoulder joints of handball players and volleyball players, ankle joints to basketball players, or, for example, to the knee joints of football players (Satrapová \& Nováková, 2012; Wilk et al., 2002).

\section{OBJECTIVES OF THE WORK}

Because of frequent functional disorders in the shoulder joint of the dominant upper extremity in volleyball and handball players, we were interested in the question of whether the functional status of the glenohumeral joint in players without difficulties shows common features. The objective of this study was to elucidate whether volleyball and handball players, experience increased ROM and specific local hypermobility in their shoulder joints already in junior categories. These changes are going to be described by trigonometric measurements and hypermobility tests according to Sachse (Lewit, 2009) and Beighton (Beighton et al., 2011). The results are monitored with respect to gender and sport specialization.

\section{METHODS}

Data collection was performed using an anamnestic questionnaire, goniometric measurement with a goniometer with a digital display, hypermobility of scapulohumeral joint according to Sachse and Beighton score. An anamnestic questionnaire was developed for the appropriate selection of participants. The measurement always took place according to the prescribed positions and according to the prescribed fixation from the literature, all lége artis. All players and the members of the control group were measured after basic athletic warm-up and a dynamic stretching for the shoulder area with the assumption of obtaining the usual functional ROM in the shoulder joint used in training or competition. All subjects were measured by one physiotherapist. The time schedule for data collection took place in November 2019.

It was a deliberate selection of participants. Athletes were selected according to the anamnestic questionnaire based on the following facts: required junior category, training or match at least 3 times a week. Participant had to play the extraleague or the 1st league of his/her category.

The anamnestic questionnaire was used to reduce the variability of the research group - the athlete should have no previous problems with the shoulder joint, such as dislocations, soft tissue damage, frozen shoulder, impingement, postoperative conditions similar disorders. All subjects included in the research group signed informed consent and the project was approved by the ethics committee FTVS UK 
(N. 149/2019). The total number of overhead athletes in the research group was 73 (there were 33 men and 40 women, 34 handball players and 39 volleyball players). 22 participants were measured in the control group (11 men and 11 women). The group includes the majority of right-handers $-91 \%$, with $9 \%$ left-handers.

\section{Data collection method}

\section{Beighton score assessment}

To distinguish whether it is only local hypermobility or generalised mobility, the Beighton score was used. Testing is performed on both halves of the body and it is possible to get 0-9 points (Beighton et al., 2011).

\section{Sachse scapulohumeral joint hypermobility test}

The test is performed sitting. We perform passive abduction of the examined arm while fixing the scapula and the collar bone from above. The range " $\mathrm{A}$ " is up to $90^{\circ}$, the range " $\mathrm{B}$ " from $90^{\circ}$ to $110^{\circ}$ and the range " $\mathrm{C}$ " above $110^{\circ}$ (Lewit, 2009).

\section{Goniometry-ROM in the shoulder joint}

During the research, the active and passive ROM in the shoulder joint was measured. The measurement was performed in all planes of the shoulder joint (Norkin \& White, 2016). That is, in the sagittal plane (extension and flexion), vertical (abduction and adduction), transverse (abduction and adduction) and in rotations (external and internal). A two-arm goniometer with a digital display was used for the measurement.

\section{Data processing}

The measured values were converted into a spreadsheet in Excel. Trigonometric values were written in degrees. Sachse hypermobility tests were recorded by a numerical record, where the test result " $\mathrm{A}$ " corresponds to the numerical value 1 , the test result " $\mathrm{B}$ " corresponds to the numerical value 2 and the test result " $\mathrm{C}$ " corresponds to the numerical value 3 . This record was chosen due to the necessary statistical processing.

Overhead athletes were then compared using statistical methods against hypotheses compared to the control group. This was followed by a comparison of the differences in the dominant shoulder between women and men, then between handball players and volleyball players, and in the end the dominant shoulder joint was compared in measured values against the non-dominant shoulder joint in all participants.

\section{Data analysis}

The R mathematical system (Free Software) was used for data analysis and statistical processing, and the R Studio environment was used, which was used for data analysis, prognostic analysis and hypothesis testing.

The Shapiro-Wilk test used on smaller populations was used to test the normality of the data. Due to the abnormality of half of the variables, non-parametric tests were used for statistical testing ( $\mathrm{p}<0.05=$ level of statistical significance). 


\section{RESULTS}

\section{File characteristics}

95 people without a prior injury of a shoulder joint in the age from 16 to 19 years old including 73 volleyball and handball players as overhead athletes were tested. There were 33 men, 40 women, 34 handballers, and 39 volleyballers. In the control group, there were 22 participants, of which 11 men and 11 women. The $91 \%$ of the study participants were right-handed. Only $9 \%$ people were left-handed.

\section{Beighton score assessment}

Beighton's score results show that the experimental and control group are balanced. Graph 1 shows both examined groups according to the degree of generalised hypermobility and thus emphasizes the balance of both groups in this score.

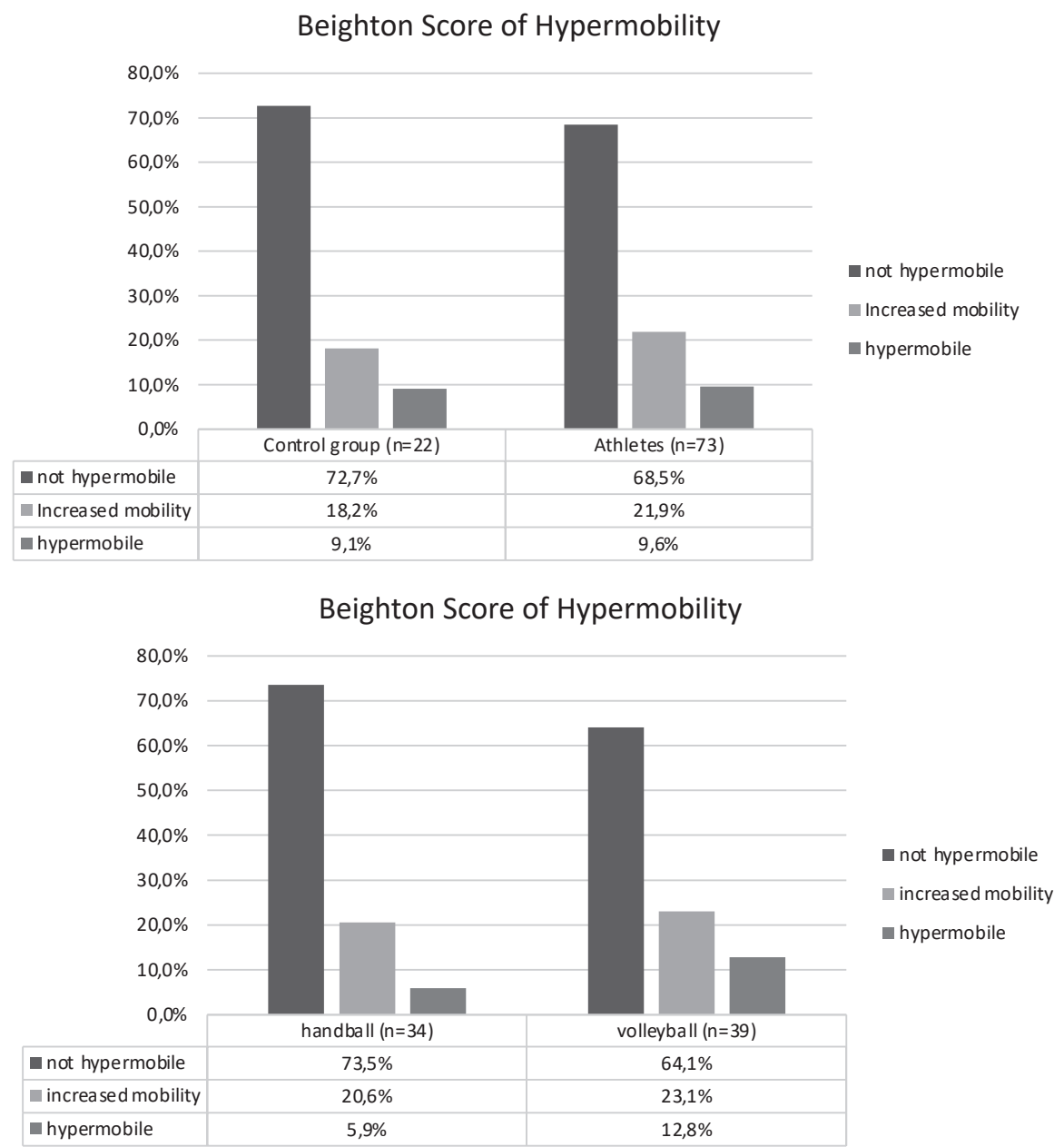

Graph 1 Evaluation of Beighton score according to the degree of generalised hypermobility a) in control group and overhead athletes and $b$ ) in handball and volleyball players (expressed as a percentage) 


\section{Sachse scapulohumeral joint hypermobility test}

Local hypermobility was considered significant if the subject did not have generalized hypermobility. Graph 2 shows the results of the scapulohumeral examination according to Sachse. It can be seen that the control group is over-represented by people with an "A" rating (no hypermobility). People with a rating of " $\mathrm{B}$ " and "C" do not even make up half of those tested, $40.9 \%$ to be precise. In overhead athletes, individuals with a physiological rating of "A" are only $4.1 \%$ and, conversely, a rating of " $\mathrm{C}$ ", the highest possible hypermobility rating for this test, was found in $79.5 \%$ of overhead athletes.

\section{Scapulohumeral joint hypermobility according to Sachse - dominant arm}

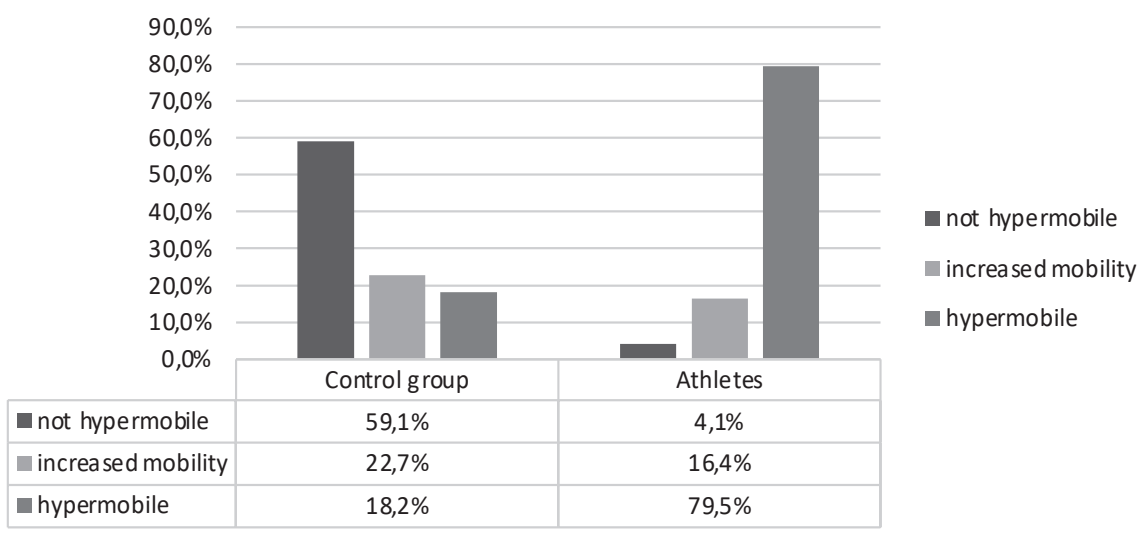

Scapulohumeral joint hypermobility according to Sachse nondominant arm

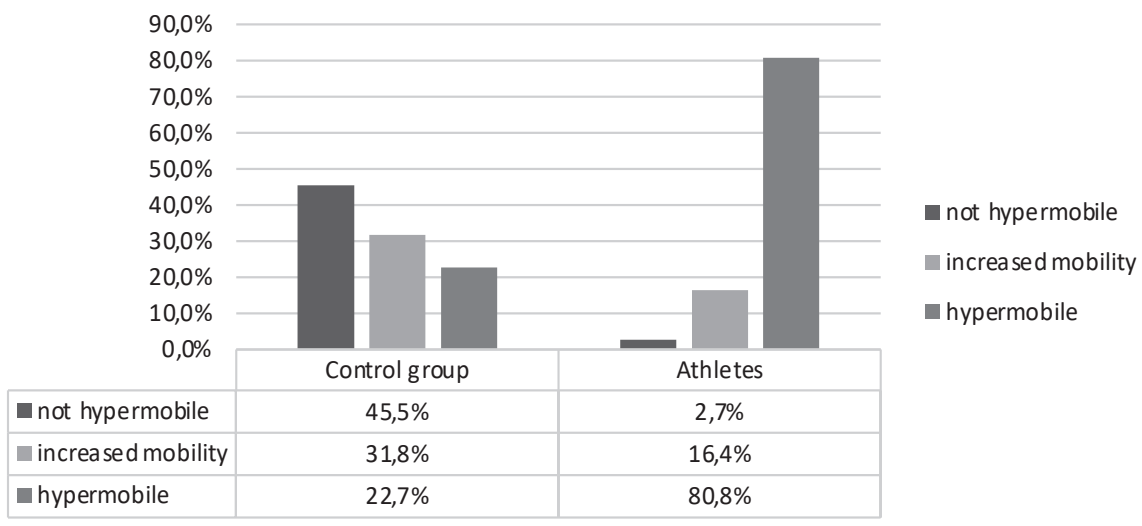

Graph 2 Evaluation of scapulohumeral joint hypermobility according to Sachse in control group and overhead athletes in a) dominant arm and b) non dominant arm (expressed as a percentage) 


\section{ROM in compare of dominant and non-dominant arm in overhead athletes}

Table 1 shows statistically significant difference in ROM in the shoulder joint of the dominant arm between overhead athletes and the control group. Measurements were performed in active and passive ROM, and comparisons and results from active ranges are included in the paper. No significant difference was observed for passive ROM.

Table 1 Comparison of shoulder joint active ROM of overhead athletes and control groups

\begin{tabular}{l|c|c}
\hline Dominant arm & P-value & Result \\
\hline Sagittal plane - Extension & 0.002 & ROM athletes increased \\
\hline Sagittal plane - Flexion & 0.121 & ROM athletes not increased \\
\hline Vertical plane - Abduction & 0.190 & ROM athletes not increased \\
\hline Transverse plane - Adduction & 0.100 & ROM athletes not increased \\
\hline Transverse plane - Abduction & 0.123 & ROM athletes not increased \\
\hline Rotation - External Rotation & 0.000 & ROM athletes increased \\
\hline Rotation - Internal Rotation & 1.000 & ROM athletes not increased \\
\hline
\end{tabular}

Table 2 compares the difference in ROM between the dominant and non-dominant arm in the examined group of overhead athletes. The difference and thus the asymmetry was found in the extension and external rotation.

Table 2 Comparison of dominant and non-dominant shoulder joint in active ROM movement in overhead athletes overhead athletes

\begin{tabular}{l|c|c}
\hline Athletes & P-value & Result \\
\hline Sagittal plane - Extension & 0.034 & asymmetry in ROM \\
\hline Sagittal plane - Flexion & 0.366 & no asymmetry \\
\hline Vertical plane - Abduction & 0.091 & no asymmetry \\
\hline Transverse plane - Adduction & 0.416 & no asymmetry \\
\hline Transverse plane - Abduction & 0.300 & no asymmetry \\
\hline Rotation - External Rotation & 0.000 & asymmetry in ROM \\
\hline Rotation- Internal Rotation & 0.326 & no asymmetry \\
\hline
\end{tabular}

Extension in the sagittal plane (see Graph 3) was more pronounced in non-dominant shoulder joints of overhead athletes.

Another direction of movement, where a significant difference between the dominant and non-dominant arm of overhead athletes was discovered, was external rotation. External rotation had a higher extent in the dominant shoulder joint of overhead athletes. As can be seen in Graph 4, both shoulder joints of overhead athletes exceed the physiological limit of $90^{\circ}$ in external rotation. The extent of the non-dominant arm is slightly above this limit, the dominant arm exceeds the limit by $9^{\circ}$. There are also some participants in the graph whose ranges of external rotation are very different 


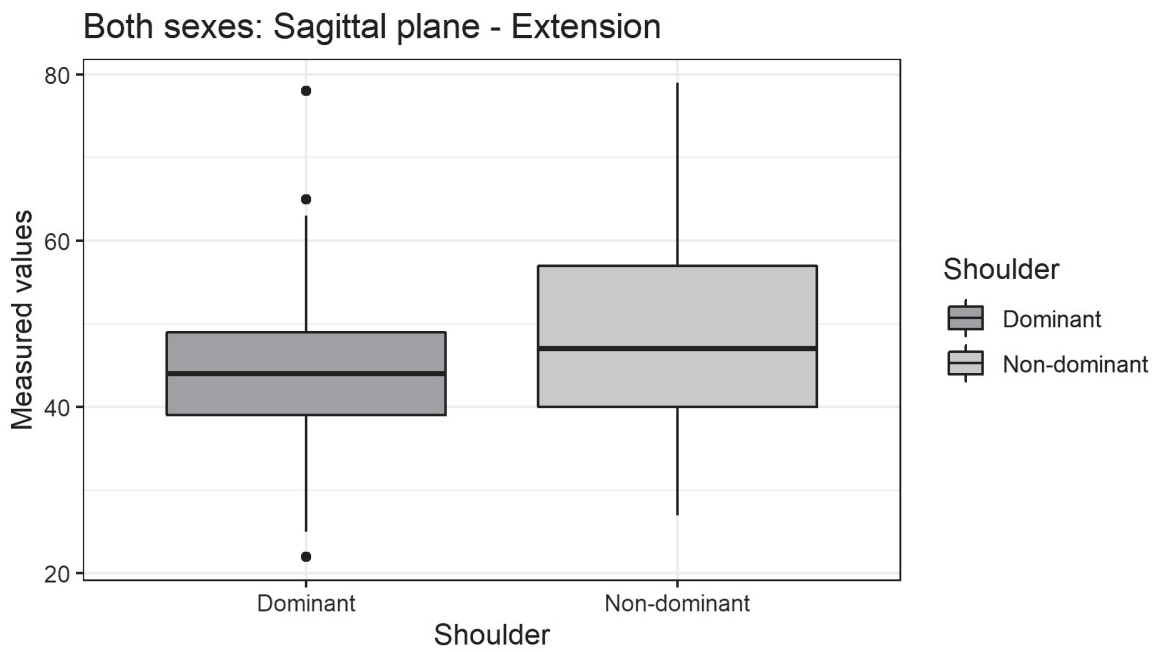

Graph 3 Extension of dominant and non-dominant shoulder joint in overhead athletes (given in degrees)

Both sexes: Rotation - External rotation

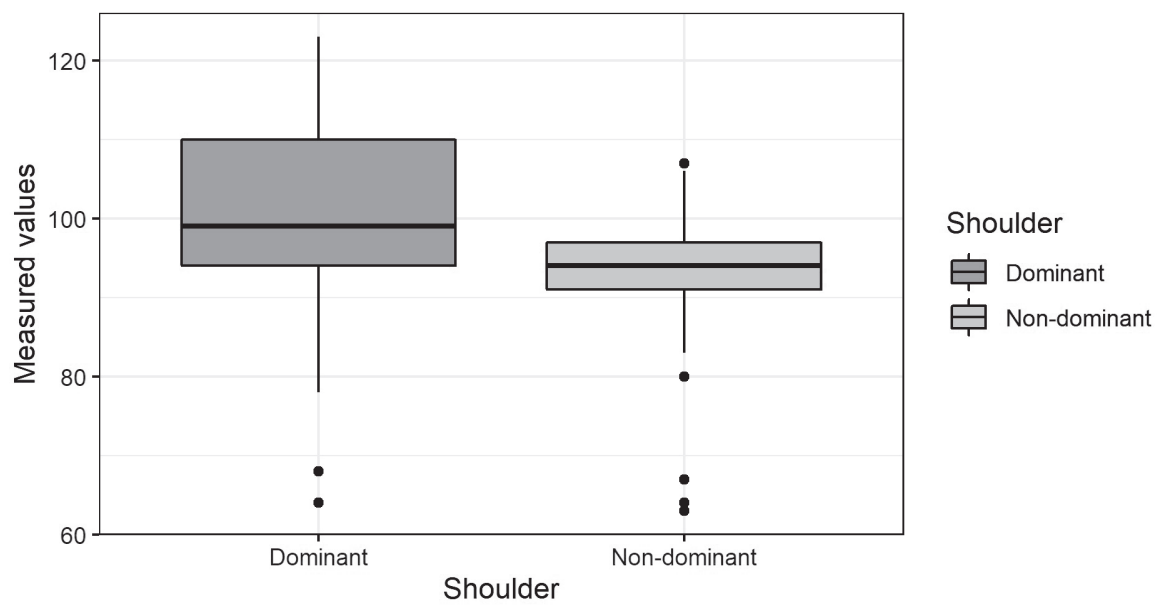

Graph 4 External rotation of the dominant and non-dominant shoulder joint in overhead athletes (given in degrees)

from the rest of the measured set. These participants are highlighted by a black dot in the graph, and with the exception of one case of a non-dominant arm, these were reduced ranges of motion in external rotation. Despite these differences, the difference between the shoulder joints is significant.

Our study showed that females compared to males playing overhead sports have significantly higher internal rotation rates of both dominant and non-dominant arms (Table 3). 
Table 3 Comparison dominant and non-dominant shoulder joint in active ROM in overhead athletes according to gender

\begin{tabular}{l|c|c}
\hline Athletes - dominant arm & P-value & Result \\
\hline Sagittal plane - Extension & 0.786 & no difference \\
\hline Sagittal plane - Flexion & 0.408 & no difference \\
\hline Vertical plane - Abduction & 0.387 & no difference \\
\hline Transverse plane - Adduction & 0.075 & no difference \\
\hline Transverse plane - Abduction & 0.357 & no difference \\
\hline Rotation - External Rotation & 0.094 & no difference \\
\hline Rotation - Internal Rotation & 0.000 & significant difference ROM \\
\hline Athletes - nondominant arm & P-value & Result \\
\hline Sagittal plane - Extension & 0.202 & no difference \\
\hline Sagittal plane - Flexion & 0.552 & no difference \\
\hline Vertical plane - Abduction & 0.481 & no difference \\
\hline Transverse plane - Adduction & 0.070 & no difference \\
\hline Transverse plane - Abduction & 0.594 & no difference \\
\hline Rotation - External Rotation & 0.859 & no difference \\
\hline Rotation - Internal Rotation & 0.000 & significant difference ROM \\
\hline
\end{tabular}

\section{DISCUSSION}

Both volleyball and handball participants showed an increased ROM to extension in the sagittal plane. The increased range may be due to the nature of the start of the spike in volleyball. Here, the emphasis is on maximum engagement in the shoulder joints with extended elbow joints, so that subsequently the arms during flexion in the shoulder joints help to make the maximum vertical jump and thus the attacker obtains optimal conditions for attack. According to the literature, handball shows the upper arc of the stretch when throwing the ball. This is the most common way and the fastest way to throw the ball. Very often, however, in handball players you can see the ball leading the so-called lower arc, when the dominant arm with the ball reaches maximum extension, in modern handball, when thrown up from the lower arc, recedes, especially due to the length of holding the ball. In handball, there is also a method of shooting so-called shot and undershot. When shooting with a shot or undershot, the dominant arm with the ball during the stretch phase gets back to maximum extension (Šibila, Pori, \& Bon, 2003).

Due to the nature of volleyball and handball, a significant burden is placed on external rotation. Wilk et al. (2011) described that there is a significant difference in the ROM between the external and internal rotators on the dominant shoulder joint. This author described that overhead athletes usually have a full ROM, but on the dominant arm the external rotation is increased by $10^{\circ}$ to $15^{\circ}$ and the internal rotation is reduced by $10^{\circ}$ to $15^{\circ}$ compared to the non-dominant arm. The results of this research agree with this statement. 
The scarf test showed an increased range of the control group compared to overhead athletes, the difference was clear between the rating "A", ie the physiological range, and the rating " $\mathrm{B}$ " mild hypermobility. However, this test is not only affected by the hypermobility of the shoulder joint, but especially by muscle hypertrophy (mainly pectoralis major and biceps humeri) and also by muscle tension, which is significantly higher in athletes than in the control group not performing any sport at the performance level. For this reason, the control group appears to be more hypermobile in this test.

A comparison of the symmetry of the ROM of the shoulder joints in the Sachse arm test was also performed. When inserted into the created scale and graphical comparison, it can be seen that the group of overhead athletes has, according to this measurement, significantly greater asymmetry between the shoulder joints than the control group. In athletes, 10 participants received a " $\mathrm{D}$ " rating, the highest degree of asymmetry in this test. In the control group, this evaluation was not achieved in any of the participants. This test confirms the difference in the ROM of the dominant arm between the athletes and the control group, but also between the dominant and non-dominant arm of the overhead athletes. When testing hypermobility of joints in overhead athletes with a control group on a Telos device, Borsa did not report any difference between these groups. The results of our research do not agree with this statement (Borsa et al., 2005).

Examination of the scapulohumeral joint is the most important test for this study in terms of hypermobility. The results of this research agree with the statement of Wilk and Arrigo (1992) that overhead athletes have a significant laxity of the scapulohumeral joint. These authors mentioned the hypermobility of this joint in overhead athletes as a "thrower's laxity". In the Sachse scapulohumeral joint test, the phenomenon of muscle tension mentioned in the scarf test can be eliminated. This test showed that overhead athletes are highly hypermobile in the scapulohumeral joint. $79 \%$ of overhead athletes had the highest " $\mathrm{C}$ " rating. In contrast, only $5 \%$ had a physiological range. In contrast, the control group had an absolute majority (59\%) with a physiological score of "A". From my point of view, this test is the most accurate evaluation, it targets exactly the given joint and the result of the examination cannot be significantly skewed, because the therapist actively participates in the examination with a clearly defined fixation.

The Beighton score was also measured when testing participants. According to the scale of this testing, it can be said that both sets were balanced in terms of generalised hypermobility.

The results show that the increased ranges of motion in extension and external rotation in goniometric measurements, as well as the increased frequency of hypermobility in the scapulohumeral joint is caused by the practice of overhead sport and is not caused by generalised hypermobility of the individual.

When comparing the ranges of movement of the dominant arms of athletes performing handball and volleyball, there was no significant difference.

When comparing the dominant and non-dominant arms in our study in overhead athletes, it was confirmed that the ranges of movement of the dominant and non-dominant arms differ significantly statistically. Compared to the Seabra study, however, the ROM in other directions differs in our study, namely in extension in the sagittal plane 
and in external rotation (Seabra et al., 2017). The increased active ROM to external rotation coincides with the Saccol study from 2016 (Saccol et al., 2016).

Extension in the sagittal plane was more pronounced in non-dominant shoulder joints. At first glance, this is a surprising measurement result, which in overhead athletes was caused mainly by volleyball participants. In my opinion, the result is caused by the stereotype of a smashing step. During the smash run, both arms, as already mentioned, get to the maximum extension, then the arms swing into the flex to allow the maximum vertical jump and the player obtains optimal conditions for attack. At the moment of contact with the ball, the dominant shoulder joint must stabilize more due to the impact of the hand on the ball. The non-dominant shoulder joint in a volleyball attack may not have this need and for this reason the non-dominant shoulder joint has a greater ROM in this direction.

External rotation was increased in the dominant shoulder joints. To achieve game goals in volleyball and handball, the shoulder dominant joint gets into extreme positions in external rotation compared to the non-dominant joint, so the ROM in this direction between the shoulders is different.

\section{CONCLUSION}

The altered ROM of the dominant arm of the sports group in two directions was demonstrated. Furthermore, local hypermobility of the scapulohumeral joint, asymmetry of the ROM of the shoulder joints and the Beighton score for generalised hypermobility were demonstrated in athletes, which was represented in both groups evenly.

The comparison of female and male athletes in the active ranges of movement measured goniometrically turned out in such a way that women showed a higher significant range in the internal rotation of the dominant and non-dominant arm.

The comparison between volleyball and handball participants turned out without a statistically significant difference. Thus, essential for a physiotherapist regarding the ROM of the shoulder joints is the fact that the athlete performs an overhead sport, in this case a ball overhead sport, rather than a specific sport.

In the last part, the work was devoted to comparing the dominant and non-dominant arms in each participant, ie athletes, but also in the control group. No significant difference in movement between the arms was found in the control group. In the group of athletes, there was a significant difference in extension in the sagittal plane and in external rotation. A higher extent was measured in the extension in the non-dominant arm and in the external rotation in the dominant arm.

\section{Author contributions}

$\mathrm{PB}, \mathrm{TN}$ and $\mathrm{LZ}$ contributed to the initial development of research, its preparation and implementation, PB led the measurements and contributed to statistical analysis.

\section{Institutional review board statement}

The study was conducted according to the guidelines of the Declaration of Helsinki, and approved by Ethics Committee (EC of Charles University, FTVS, EK 149/2019). Informed Consent Statement: Informed consent was obtained from all subjects involved in the study. 


\section{REFERENCES}

Beighton, P. H., Grahame, R., \& Bird, H. (2011). Hypermobility of joints. Springer Science \& Business Media.

Bigliani, L. U., Codd, T. P., Connor, P. M., Levine, W. N., Littlefield, M. A., \& Hershon, S. J. (1997). Shoulder motion and laxity in the professional baseball player. The American Journal of Sports Medicine, 25(5), 609-613.

Borsa, P. A., Wilk, K. E., Jacobson, J. A., Scibek, J. S., Dover, G. C., Reinold, M. M., \& Andrews, J. R. (2005). Correlation of range of motion and glenohumeral translation in professional baseball pitchers. The American Journal of Sports Medicine, 33(9), 1392-1399.

Janda, V. (2004). Muscle function tests (in Czech). Prague: Grada Publishing.

Jobe, F. W., \& Pink, M. (1993). Classification and treatment of shoulder dysfunction in the overhead athlete. Journal of Orthopaedic \& Sports Physical Therapy, 18(2), 427-432.

Lewit, K. (2009). Manipulative therapy: Musculoskeletal medicine. Elsevier Health Sciences.

Norkin, C. C., \& White, D. J. (2016). Measurement of joint motion: a guide to goniometry. F.A. Davis.

Pappas, A. M., Zawacki, R. M., \& Sullivan, T. J. (1985). Biomechanics of baseball pitching: A preliminary report. The American Journal of Sports Medicine, 13(4), 216-222.

Russek, L. N. (1999). Hypermobility syndrome. Physical therapy, 79(6), 591-599.

Saccol, M. F., Almeida, G. P. L., \& de Souza, V. L. (2016). Anatomical glenohumeral internal rotation deficit and symmetric rotational strength in male and female young beach volleyball players. Journal of Electromyography and Kinesiology, 29, 121-125.

Sachse, J., \& Janda, V. (2004). Constitutional hypermobility: a review. Manuelle Medizin, 42(1), 33-40.

Satrapová, L., \& Nováková, T. (2012). Hypermobility in sport (in Czech). Rehabilitace a fyzikální lékařství, 19(4), 199-202.

Seabra, P., Van Eck, C. F., Sá, M., \& Torres, J. (2017). Are professional handball players at risk for developing a glenohumeral internal rotation deficit in their dominant arm?. The Physician and Sportsmedicine, 45(2), 77-81.

Šibila, M., Pori, P., \& Bon, M. (2003). Basic kinematic differences between two types of jump shot techniques in handball. Universitatis Palackianae Olomucensis Gymnica, 33(1), 19-26.

Wilk, K. E., \& Arrigo, C. (1992). An integrated approach to upper extremity exercises. Orthopaedic Physical Therapy Clinics of North America, 1(2), 337-360.

Wilk, K. E., Macrina, L. C., Fleisig, G. S., Porterfield, R., Simpson, C. D., Harker, P., ... \& Andrews, J. R. (2011). Correlation of glenohumeral internal rotation deficit and total rotational motion to shoulder injuries in professional baseball pitchers. The American Journal of Sports Medicine, 39(2), 329-335.

Wilk, K. E., Meister, K., \& Andrews, J. R. (2002). Current concepts in the rehabilitation of the overhead throwing athlete. The American Journal of Sports Medicine, 30(1), 136-151. 